Lexique vivant de la révolution et de la guerre en Syrie

\title{
Disqualifier ses opposants \\ politiques pour mieux les \\ éradiquer : l'exemple de \\ jarâthîm (bactéries)
}

Nisrine Al-Zahre

Published on: Jun 24, 2020

DOI: $10.21428 / 3633 f a e 9 . e 54 c 11 b d$

License: Creative Commons Attribution-NonCommercial-NoDerivatives 4.0 International License (CC-BY-NC-ND 4.0). 
Cet article a été initialement publié sur le carnet de recherche du programme de recherche ANR Shakk (Projet-ANR-17-CE26-0021) le 24 juin 2020 : https://shakk.hypotheses.org $/ 2177$

\section{Visit the web version of this article to view interactive content. ما علاقة قضاء الجيش السوري على "جراثيم" بالكورونا؟ \\ “L’armée arabe syrienne a purifié la terre syrienne de nombreuses bactéries”. Extrait du discours du ministre de la Santé syrien du 10 Mars 2020}

Le 10 mars 2020, le ministre de la Santé syrien est interrogé par la chaîne d'informations officielle, concernant les chiffres des malades atteints du virus covid 19. Il répond en faisant l'éloge de l'armée qui « salvatrice » aurait « purifié » le sol syrien de ses « bactéries : jarâthîm ». Le ministre ne fait que « répéter » et « citer » $\underline{1}$ les expressions que Bachar Al Assad a lui-même utilisées neuf ans plus tôt en juin 2011, durant son deuxième discours, prononcé au siège du rectorat de l’Université de Damas. Le président qualifie alors les événements de «complot », et ceux qui y prennent part de bactéries (m.7 :52) « qui se multiplient à chaque instant et partout. » Il poursuit en ces termes : "Il serait difficile de les anéantir complètement, mais on pourrait néanmoins fortifier le système immunitaire pour les combattre [...] Ainsi il faut repérer les failles dans " notre » système immunitaire qui permettent aux bactéries de s'infiltrer dans nos corps. [...] Les bactéries existent partout, sur la peau, dans les intestins et il ne sert à rien de vouloir les éliminer définitivement, il suffit de rendre notre système de défense plus immune ». Dans ce même discours prononcé à l’Université de Damas, le président s'emploie à catégoriser les personnes qui participent aux manifestations, ou autrement dit celles et ceux qui constituent « les failles dans le système immunitaire », auxquelles il faudrait « remédier ». Selon lui, « il y a une majorité de personnes qui ont des vraies revendications, et pour lesquelles il faut avancer des solutions concrètes ; cette majorité est une composante patriotique (mukawwin watanî) dont les revendications se situent sous le plafond de la patrie (taht saqf al-watan) et elle n'est pas subordonnée à un agenda extérieur. La deuxième catégorie est une minorité de saboteurs qui crée le chaos. La troisième catégorie est la plus minoritaire, mais la plus dangereuse, il s'agit d'extrémistes fanatiques dont l'idéologie est «takfiriste»2. Cette « composante » essaye de s'infiltrer en Syrie depuis des décennies. Masquée, elle cible le chaos et la destruction sous le nom de la liberté. 
Elle est payée par des agendas extérieurs, elle essaye de défigurer l'image de la patrie et d'affaiblir sa position... [...]».

Depuis le début des révolutions dans le monde arabe, les expressions déshumanisantes se bousculent sur les lèvres de plusieurs dirigeants : « rongeurs : qawârid», « cafards : sarâsir », «infiltrés : mundasîn », « racaille : huthâla », " traîtres : khawana », « agents : 'umalâ'», etc. Un fait important est pourtant à noter dans le discours de Bachar Al Assad, c'est que le mot « watan : patrie » a été prononcé plus qu'une trentaine de fois. Cette juxtaposition permanente entre, d'une part, la patrie et le patriotisme, et, d'autre part, les bactéries et la minorité dangereuse n'est pas sans intérêt, j'essayerai de l'interpréter plus tard dans cette notice.

On pourrait se poser la question de savoir si les propos du ministre de la Santé, après tant d'années de « répétitions » et de « citations » insultantes, réussissent encore à susciter des réactions chez les Syriens, même chez ceux qui sont loin de Damas à des milliers de kilomètres ? Il est évident que ce cynisme, inapproprié par rapport à un contexte d'urgence sanitaire, rappelle amèrement le clivage permanent entre les faits et la réponse des autorités à ces mêmes faits. Le non-contexte d'un énoncé, ou le contexte inapproprié à un énoncé est un élément d'étrangeté, profondément déstabilisant, pour reprendre la définition de la félicité d'un acte de langage du philosophe John Austin $\underline{3}$.

Mais au-delà de la perturbation et de la brisure du contexte, pour chaque individu la blessure reflète des strates plus profondes et plus anciennes : être insulté, « being called a name» $\underline{4}$ est non seulement douloureux, mais générateur d'un sentiment paralysant d'être abject $\underline{5}$ et susceptible d'éradication.

Salwa Ismail $\underline{6}$ considère l'abjection comme un mécanisme de gouvernance et d'assujettissement des individus par le régime syrien, qui va au-delà d'une simple réaffirmation des rapports de pouvoir entre dominés à dominants. Rendre des personnes « abjectes » pour mieux s'assurer le soutien du reste de la population, sert d'autres objectifs : c'est un mécanisme qui permet de prévenir et de défaire toutes subjectivités oppositionnelles.

Les répercussions du pouvoir discursif des mots s'insinuent partout : tel un lexique imposé, on les retrouve jusque dans les discours de personnalités par ailleurs insignifiantes en termes de décisions politiques, comme le ministre de la Santé. Il est évident que l'efficacité de ces termes déshumanisants est assurée par leur constante 
répétition, dans le sens où le mécanisme de l'abjection consiste à mettre en échec en permanence des potentiels oppositionnels dans les subjectivités.

Arrêtons-nous sur l'expression anglaise « being called a name » analysée par Judith Butler. Empruntant à Althusser la notion d' « interpellation »; à Austin celle d'effets « perlocutoire » et « illocutoire » des actes du langage et à Bourdieu celle d' « habitus » corporel, Butler explique que le «name/insulte »n'a son pouvoir illocutoire performatif et douloureux que dans la mesure où il y a une complicité sociale entre tous les acteurs discursifs, y compris la personne ciblée par la parole haineuse, uniquement dans la mesure où les « répétitions » et les «citations » sont perpétuées inconsciemment et intériorisées dans les habitus corporels. La blessure corporelle causée par la parole n'est explicable que par l'épaisseur de l'historicité du terme utilisé et la disparition de la figure/ signature de celui qui en est à l'origine. Elle se comprend par la diffusion de la haine. Toujours selon Butler, casser la performativité du langage du pouvoir qui « insulte » ne serait possible que par la réappropriation de l'insulte, comme acte de résistance qui briserait le circuit de la circulation et redéfinirait les codes sémantiques, comme ce fut le cas avec l'appellation « queer » qui a été réappropriée et redéfinie par les communautés LGBT de manière à en faire un symbole d'autodétermination et de libération plutôt qu'une insulte.

Dans le cas syrien, on a pu observer des tentatives de réappropriation des étiquettes/insultes à caractère exclusivistes $\underline{7}$, afin de subvertir leurs sémantiques et y résister. Pour autant, malgré ces efforts de subversion, il semble que ces insultes continuent d'être efficaces : d'avoir un impact émotionnel abaissant, voire destructeur sur celles et ceux auxquels elles s'adressent. Dans ce cas de figure, il devient impossible de briser le cercle vicieux instauré par le pouvoir qui consiste à « interpeller » les individus par une identité qui n'est pas la leur. Derrière ce pouvoir des mots, il y a probablement le fait que politiquement les opposants au régime se trouvent dans une position de défaite qui crédibilise/authentifie le discours insultant du vainqueur. Cependant, l'effet perlocutoire négatif pourrait également se trouver ailleurs. Dans le discours de Bachar Al-Assad cité précédemment, il se situe dans la juxtaposition permanente entre, d'une part « jarâthîm : bactéries » - et ses variantes déshumanisantes « khâ'in : traître », « 'amîl : agent »- et, d'autre part, « watan : patrie $»$. . Cette juxtaposition transforme les insultes en variantes de «khâ'in ». De plus, l'acte qui consiste à rendre un sujet abject est un geste d'exclusion de la « patrie » envers laquelle l'individu est toujours redevable, mais dans laquelle la citoyenneté est absente et surtout qui représente une référence affective pesante, vague et fragilisante en raison des menaces permanentes de bannissement. Il est 
difficile, voire pénible pour l'opposant qui est par définition en quête d'une légitimité politique de s'approprier le qualitatif de « traître » ou de « patriote défaillant », même pour en jouer. Un tel acte ne serait possible qu'au prix d'une redéfinition de la notion de « patrie ». Se réapproprier le qualificatif de «traître » ne peut être vécu comme un acte de résistance ou comme la première étape vers la redéfinition sémantique du terme watan. S'y risquer, serait perçu comme un acte cynique, voir grotesque. Encore une fois, en opérant une équivalence entre « bactéries » et «traites à la patrie », le pouvoir tire les ficelles de l'exclusion et de la délégitimation. L'entreprise de déshumanisation à l'œuvre ici n'a pas seulement pour objectif de rendre possibles des crimes de masse, elle vise surtout le politicide en jetant l'opprobre sur les rebelles désignés comme « les traîtres qui osent poignarder la patrie dans le dos ».

Une question reste en suspens : qui, en 2020, est la cible des insultes du régime après l'annonce officielle de la «purification : tathîr » de la Syrie de ses sujets abjects et de son « homogénéisation : tajânus) ? S'agirait-il, non pas d'insultes, mais de menaces adressées à ceux qui, restés en Syrie, pourraient à leur tour devenir « abjects » et « traîtres »? Le régime, serait-il enfermé dans ses répétitions discursives, incapable de s'adresser à sa population autrement ? Ou peut-être est-il simplement prisonnier de ses sujets insultés sans lesquels il perdrait son emprise et une grande part de son pouvoir.

\section{Bibliographie}

La bibliographie de cet article est disponible dans le groupe Zotero du Lexique (https://www.zotero.org/groups/4550572/syria lexicon/collections/2J7BHLSA).

\section{Footnotes}

1. Butler, Judith. Excitable Speech. 0 éd. Routledge, 2013.

https://doi.org/10.4324/9780203948682.

2. «Takfiriste : takfîrî » du mot «takfir : excommunication ». Sont ainsi désignés les islamistes radicaux qui considèrent les musulmans ne partageant pas leur point de vue comme étant des apostats et donc des cibles légitimes. Les autorités syriennes ont dès les premiers mois de la révolte utilisé ce terme, ainsi que celui de « salafî: salafiste » pour désigner les manifestants quels qu'ils soient. $ヒ$

3. Austin, J. L, J. O Urmson, et Marina Sbisà. How to Do Things with Words, 1975. http://www.worldcat.org/oclc/1811317. Selon l'auteur, la félicité d'un acte de langage est l'ensemble de conditions entretenues par les acteurs d'un acte de langage pour 
une meilleure communication, parmi ces conditions il y a le respect des rituels, notamment quand il s'agit d'un énoncé performatif, l'appropriation du contenu de l'énoncé au contexte de l'énonciation, etc. L'absence du contexte approprié à l'énoncé est en soi un « contexte de perturbation », un fait expliqué par Austin et repris par Judith Butler op. cit. $\doteq$

4. Butler, Judith. Excitable Speech. Routledge, 2013. https://doi.org/10.4324/9780203948682.

5. Il est intéressant de noter que dans l'étymologie du mot, on retrouve le sens de l'éloignement et du rejet du sale, du dégoutant, un sens exploré psychanalytiquement par Julia Kristeva et utilisé plus tard dans les sciences humaines au sujet de l'ordre social pour la préservation duquel le rejet de certains individus serait indispensable. Cf. Kristeva, Julia. Pouvoirs de l'horreur: essai sur l'abjection. Paris: Éditions du Seuil, 1980. http://www.worldcat.org/oclc/299370927. $\bullet$

6. Ismail, Salwa. The Rule of Violence: Subjectivity, Memory and Government in Syria. 1 $1^{\text {re }}$ éd. Cambridge University Press, 2018. https://doi.org/10.1017/9781139424721. p. 19-21.

7. A titre d'exemple, peu de temps après le déclenchement de la révolution en Syrie, un blog de l'opposition s'est fait connaitre sur le net sous le nom d' " Infiltrée syrienne : mundassa sûriya ». Ce blog a aujourd'hui disparu, mais a été archivé sur le site Creative Memoory of the Syrian Revolution : الذاكرة الإبداعية للثورة السورية “المندسة السورية“. Consulté le 11 janvier 2022. https://creativememory.org/ar/archives/57807/\%d8\%a8\%d8\%b1\%d9\%86\%d8\%a7\%d9 \%85\%d8\%ac-\%d8\%b9\%d9\%86\%d8\%b2\%d8\%a9-\%d9\%88\%d9\%84\%d9\%88\%d8\%b7\%d8\%a7\%d8\%b1\%d8\%aa-\%d8\%af\%d9\%84\%d9\%8a\%d9\%84/.

8. Une étude reste à faire sur les familles de désignation (organiques (jarâthîm), religieuses (takfîrî) et politiques (khâ'in)) pour comprendre comment elles s'agencent et à quels moment elles sont mobilisées dans le discours présidentiel. $\subseteq$ 